\title{
Feasibility of Experimental Realization of Entangled Bose-Einstein Condensation
}

\author{
Europhys. Lett. 86 (2009) 60008
}

\author{
YU SHI ${ }^{(a)}$ \\ Department of Physics, Fudan University, Shanghai 200433, China \\ Kavli Institute for Theoretical Physics China at the Chinese Academy of Sciences, Beijing 100080, China \\ PACS 03.75. Mn - \\ PACS 03.75.Gg -
}

\begin{abstract}
We examine the practical feasibility of the experimental realization of the so-called entangled Bose-Einstein condensation (BEC), occurring in an entangled state of two atoms of different species. We demonstrate that if the energy gap remains vanishing, the entangled BEC persists as the ground state of the concerned model in a wide parameter regime. We establish the experimental accessibility of the isotropic point of the effective parameters, in which the entangled BEC is the exact ground state, as well as the consistency with the generalized Gross-Pitaevskii equations. The transition temperature is estimated. Possible experimental implementations are discussed in detail.
\end{abstract}

Recently, a novel type of Bose-Einstein condensation (BEC), characterized by an entangled order parameter, herein simply called entangled BEC, was found to be the ground state of a mixture of two species of atoms with spins [1]. The entangled BEC, which occurs in an interspecies two-particle entangled state, is interesting in several aspects. First, it is not a mean field state of single atoms, unlike the usual multicomponent BEC, whose ground state is simply a direct product of wave functions of all the atoms, with each component described by a same wave function. Second, the interspecies twoparticle entangled state bears some similarity to Cooper pairing of fermions, but it is not a weakly bound state. In other words, the entangled order parameter is nonlocal. Third, this entangled condensate may serve as a source of Einstein-Podolsky-Rosen pairs, the key components in a lot of quantum information processes. The nonlocal nature of the order parameter makes it convenient to be used for this purpose, if experimentally realized. Fourth, as elaborated below, the entangled BEC is a new kind of fragmented condensation, i.e., with macroscopic occupation of more than one single-particle state [2], which has attracted a lot of attention in recent years [3,4].

The entangled BEC was previously established as the

(a)Email: yushi@fudan.edu.cn ground state of the concerned model, when the effective parameters are so chosen that the total pseudospin of the system is conserved, in consistency with the common wisdom that fragmentation is due to spin symmetry [4]. An important question is how this ground state can survive in a wide parameter regime, where the Hamiltonian breaks or lowers the symmetry. This is crucial for experimental realization. There have been some numerical investigations on this issue demonstrating the persistence of interspecies entanglement [1]. But there has been a lack of analytical discussions. Moreover, it has not been clear enough how to actually implement the entangled BEC in experiments.

In this Letter, we investigate several related issues around the experimental realization of the entangled BEC. First we consider the concerned model with a deviation from the symmetric parameter point, where the entangled $\mathrm{BEC}$ is the exact ground state. Treating the deviation as a symmetry breaking perturbation, we find that for a finitevolume gas, if the energy gap tends to vanish, then the ground state approaches the unperturbed entangled BEC. This establishes a significant parameter regime for the entangled BEC. Afterwards, we examine the consistency between the isotropic point of the effective parameters and the generalized Gross-Pitaevskii equations of the orbital wave functions, which are in turn related to those effective parameters. After a brief estimation of the transition 
temperature, we discuss in detail how to experimentally implement the entangled BEC. In view of recent related experimental progress, we suggest that it is feasible to experimentally realize the entangled BEC in an optical trap.

The model. - Consider a dilute gas of two species (or isotopes) of bosonic atoms in a trap [1]. Each atom possesses an internal degree of freedom represented as a pseudospin with $z$-component basis states $\uparrow$ and $\downarrow$. Then the many-body Hamiltonian is $\mathcal{H}=\sum_{i=a, b}\left[\sum_{\sigma=\uparrow, \downarrow} \int d^{3} r \psi_{i \sigma}^{\dagger} h_{i \sigma} \psi_{i \sigma}+\right.$ $\left.\frac{1}{2} \int d^{3} r \psi_{i \sigma_{1}}^{\dagger} \psi_{i \sigma_{2}}^{\dagger} U_{\sigma_{1} \sigma_{2} \sigma_{3} \sigma_{4}}^{(i i)} \psi_{i \sigma_{3}} \psi_{i \sigma_{4}}\right]$

$\int d^{3} r \psi_{a \sigma_{1}}^{\dagger} \psi_{b \sigma_{2}}^{\dagger} U_{\sigma_{1} \sigma_{2} \sigma_{3} \sigma_{4}}^{(a b)} \psi_{b \sigma_{3}} \psi_{a \sigma_{4}}$, where the independent variable $\mathbf{r}$ in the field operator $\psi_{i \sigma}$, the single particle Hamiltonian $h_{i \sigma}$ and the interaction $U_{\sigma_{1} \sigma_{2} \sigma_{3} \sigma_{4}}^{(i j)}$ are all omitted for brevity. $h_{i \sigma}=-\hbar^{2} \nabla_{i}^{2} / 2 m_{i}+U_{i \sigma}$, where $U_{i \sigma}$ is the trapping potential. The s-wave effective interaction $U_{\sigma_{1} \sigma_{2} \sigma_{3} \sigma_{4}}^{(i j)}\left(\mathbf{r}_{i}-\mathbf{r}_{j}^{\prime}\right)=\left(2 \pi \hbar^{2} \xi_{\sigma_{1} \sigma_{2} \sigma_{3} \sigma_{4}}^{(i j)} / \mu_{i j}\right) \delta\left(\mathbf{r}_{i}-\mathbf{r}_{j}^{\prime}\right)$, $(i, j=a, b)$, where $\mu_{i j}=m_{i} m_{j} /\left(m_{i}+m_{j}\right)$ is the effective mass, $\xi_{\sigma_{1} \sigma_{2} \sigma_{3} \sigma_{4}}^{(i)}$ is scattering length for an allowed channel in which the initial pseudospins of $i$ and $j$ are $\sigma_{4}$ and $\sigma_{3}$, respectively, while their final pseudospins are $\sigma_{1}$ and $\sigma_{2}$, respectively. As usual, in ignorance of the depletion, the orbital degree of freedom of each atom is constrained in the manifold of the single-particle orbital ground states $\phi_{i \sigma}\left(\mathbf{r}_{i}\right)$. Hence $\psi_{a \sigma}=a_{\sigma} \phi_{a \sigma}\left(\mathbf{r}_{a}\right)$ and $\psi_{b \sigma}=b_{\sigma} \phi_{b \sigma}\left(\mathbf{r}_{b}\right)$, where $a_{\sigma}$ and $b_{\sigma}$ are annihilation operators. Under the conservation of $z$ component of the total pseudospin in each scattering, the many-body Hamiltonian is simplified to

$$
\begin{array}{r}
\mathcal{H}=\sum_{i, \sigma} f_{i \sigma} N_{i \sigma}+\frac{1}{2} \sum_{i, \sigma \sigma^{\prime}} K_{\sigma \sigma^{\prime}}^{(i i)} N_{i \sigma} N_{i \sigma^{\prime}} \\
+\sum_{\sigma \sigma^{\prime}} K_{\sigma \sigma^{\prime}}^{(a b)} N_{a \sigma} N_{b \sigma^{\prime}}+\frac{K_{e}}{2}\left(a_{\uparrow}^{\dagger} a_{\downarrow} b_{\downarrow}^{\dagger} b_{\uparrow}+a_{\downarrow}^{\dagger} a_{\uparrow} b_{\uparrow}^{\dagger} b_{\downarrow}\right),
\end{array}
$$

where $N_{i \sigma}$ is the number of atoms of species $i$ with pseudospin $\sigma, N_{i}=N_{i \uparrow}+N_{i \downarrow}$ is conserved, $f_{i \sigma} \equiv \epsilon_{i \sigma}-K_{\sigma \sigma}^{(i i)} / 2$,

$$
\epsilon_{i \sigma}=\int \phi_{i \sigma}^{*}\left(-\hbar^{2} \nabla_{i}^{2} / 2 m_{i}+U_{i \sigma}\right) \phi_{i \sigma} d^{3} r
$$

is the single particle energy of an atom of species $i$ and pseudospin $\sigma$. Note that there is no a priori requirement on the relation between $\phi_{i \uparrow}$ and $\phi_{i \downarrow}$. $K_{e}$ and $K_{\sigma \sigma^{\prime}}^{(i j)}$ are effective parameters proportional to the corresponding scattering lengths, and are defined in the following way. First, for the scattering in which an $i$-atom flips from $\sigma_{4}$ to $\sigma_{1}$ while a $j$-atom flips from $\sigma_{3}$ to $\sigma_{2}$, define

$$
K_{\sigma_{1} \sigma_{2} \sigma_{3} \sigma_{4}}^{(i j)} \equiv \frac{2 \pi \hbar^{2} \xi_{\sigma_{1} \sigma_{2} \sigma_{3} \sigma_{4}}^{(i j)}}{\mu_{i j}} \int \phi_{i \sigma_{1}}^{*} \phi_{j \sigma_{2}}^{*} \phi_{j \sigma_{3}} \phi_{i \sigma_{4}} d^{3} r .
$$

Then in accordance with the convention of Leggett [5], we use the shorthands $K_{\sigma \sigma}^{(i i)} \equiv K_{\sigma \sigma \sigma \sigma}^{(i i)}$, and $K_{\sigma \bar{\sigma}}^{(i i)} \equiv$ $2 K_{\sigma \bar{\sigma} \bar{\sigma} \sigma}^{(i i)}=2 K_{\sigma \bar{\sigma} \sigma \bar{\sigma}}^{(i i)}$ for $\sigma \neq \bar{\sigma}$ for intraspecies scattering, while $K_{\sigma \sigma^{\prime}}^{(a b)} \equiv K_{\sigma \sigma^{\prime} \sigma^{\prime} \sigma}^{(a b)}$ for interspecies scattering.
For interspecies pseudospin exchange scattering, we denote $K_{e} \equiv 2 K_{\uparrow \downarrow \uparrow \downarrow}^{(a b)}=2 K_{\downarrow \uparrow \downarrow \uparrow}^{(a b)}$. The last term of the Hamiltonian (11) represents pseudospin-exchange scattering between two atoms of different species. Without loss of generality, suppose $N_{a} \geq N_{b}$.

Ground State and fragmentation. - Using the total pseudospins of the two species,

$$
\mathbf{S}_{a} \equiv \sum_{\sigma, \sigma^{\prime}} a_{\sigma}^{\dagger} \mathbf{s}_{\sigma \sigma^{\prime}} a_{\sigma^{\prime}}, \mathbf{S}_{b} \equiv \sum_{\sigma, \sigma^{\prime}} b_{\sigma}^{\dagger} \mathbf{s}_{\sigma \sigma^{\prime}} b_{\sigma^{\prime}},
$$

and subtracting a constant, the Hamiltonian can be rewritten as

$$
\mathcal{H}=\mathcal{H}_{0}+\mathcal{H}_{1},
$$

with

$$
\begin{gathered}
\mathcal{H}_{0}=K_{e} \mathbf{S}_{a} \cdot \mathbf{S}_{b} \\
\mathcal{H}_{1}=\left(J_{z}-K_{e}\right) S_{a z} S_{b z}+B_{a} S_{a z}+B_{b} S_{b z}+C_{a} S_{a z}^{2}+C_{b} S_{b z}^{2}
\end{gathered}
$$

where

$$
\begin{aligned}
J_{z}= & K_{\uparrow \uparrow}^{(a b)}+K_{\downarrow \downarrow}^{(a b)}-K_{\uparrow \downarrow}^{(a b)}-K_{\downarrow \uparrow}^{(a b)}, \\
B_{a}= & f_{a \uparrow}-f_{a \downarrow}+\frac{N_{a}}{2}\left(K_{\uparrow \uparrow}^{(a a)}-K_{\downarrow \downarrow}^{(a a)}\right) \\
& +\frac{N_{b}}{2}\left(K_{\uparrow \uparrow}^{(a b)}+K_{\uparrow \downarrow}^{(a b)}-K_{\downarrow \uparrow}^{(a b)}-K_{\downarrow \downarrow}^{(a b)}\right), \\
B_{b}= & f_{b \uparrow}-f_{b \downarrow}+\frac{N_{b}}{2}\left(K_{\uparrow \uparrow}^{(b b)}-K_{\downarrow \downarrow}^{(b b)}\right)+ \\
& \frac{N_{a}}{2}\left(K_{\uparrow \uparrow}^{(a b)}+K_{\downarrow \uparrow}^{(a b)}-K_{\uparrow \downarrow}^{(a b)}-K_{\downarrow \downarrow}^{(a b)}\right), \\
C_{a}= & \frac{1}{2}\left(K_{\uparrow \uparrow}^{(a a)}+K_{\downarrow \downarrow}^{(a a)}-K_{\uparrow \downarrow}^{(a a)}-K_{\downarrow \uparrow}^{(a a)}\right), \\
C_{b}= & \frac{1}{2}\left(K_{\uparrow \uparrow}^{(b b)}+K_{\downarrow \downarrow}^{(b b)}-K_{\uparrow \downarrow}^{(b b)}-K_{\downarrow \uparrow}^{(b b)}\right) .
\end{aligned}
$$

$\mathcal{H}_{0}$ is symmetric under $S U(2 S+1)$, where $S$ is the total spin quantum number. The eigenstates of $\mathcal{H}_{0}$ are $\left|S, S_{z}\right\rangle$ 's, where $S=S_{a}-S_{b}, \cdots, S_{a}+S_{b}, S_{z}=-S, \cdots, S$ is the $z$-component of the total pseudospin, $S_{a}=N_{a} / 2, S_{b}=$ $N_{b} / 2$. Obviously, except the ground state in the case of $N_{a}=N_{b}$, the eigenstates are degenerate with respect to $S_{z}$.

The ground state of $\mathcal{H}_{0}$, in the sector of $S_{z}$, is

$$
\left|G_{S_{z}}\right\rangle=\mathcal{A}\left(a_{\uparrow}^{\dagger}\right)^{n_{\uparrow}}\left(a_{\downarrow}^{\dagger}\right)^{n_{\downarrow}}\left(a_{\uparrow}^{\dagger} b_{\downarrow}^{\dagger}-a_{\downarrow}^{\dagger} b_{\uparrow}^{\dagger}\right)^{N_{b}}|0\rangle,
$$

for which $S=S_{a}-S_{b}=\left(N_{a}-N_{b}\right) / 2, \mathcal{A}$ is the normalization constant, $n_{\uparrow}=N_{a} / 2-N_{b} / 2+S_{z}, n_{\downarrow}=N_{a} / 2-N_{b} / 2-$ $S_{z}, S_{z}=-\left(N_{a}-N_{b}\right) / 2,-\left(N_{a}-N_{b}\right) / 2+1, \cdots,\left(N_{a}-N_{b}\right) / 2$ is a conserved quantity.

The ground state $\left|G_{S_{z}}\right\rangle$ is a special kind of fragmented condensate. Because $a$ and $b$ are two different species of atoms, one should define a one-particle reduced density matrix for each species respectively. For species $i$, it is

$$
\rho_{i}\left(\mathbf{r}, \mathbf{r}^{\prime}\right) \equiv\left\langle G\left|\hat{\psi}_{i}^{\dagger}\left(\mathbf{r}^{\prime}\right) \hat{\psi}_{i}(\mathbf{r})\right| G\right\rangle,
$$

where

$$
\begin{gathered}
\hat{\psi}_{a}(\mathbf{r})=\hat{a}_{\uparrow} \phi_{a \uparrow}(\mathbf{r})|\uparrow\rangle+\hat{a}_{\downarrow} \phi_{a \downarrow}(\mathbf{r})|\downarrow\rangle, \\
\hat{\psi}_{b}(\mathbf{r})=\hat{b}_{\uparrow} \phi_{b \uparrow}(\mathbf{r})|\uparrow\rangle+\hat{b}_{\downarrow} \phi_{b \downarrow}(\mathbf{r})|\downarrow\rangle .
\end{gathered}
$$

It is evaluated that

$$
\begin{aligned}
\rho_{a}\left(\mathbf{r}, \mathbf{r}^{\prime}\right)= & \left(N_{a} / 2+S_{z}\right) \phi_{a \uparrow}^{*}\left(\mathbf{r}^{\prime}\right) \phi_{a \uparrow}(\mathbf{r}) \\
& +\left(N_{a} / 2-S_{z}\right) \phi_{a \downarrow}^{*}\left(\mathbf{r}^{\prime}\right) \phi_{a \downarrow}(\mathbf{r}), \\
\rho_{b}\left(\mathbf{r}, \mathbf{r}^{\prime}\right)= & N_{b} / 2 \phi_{b \uparrow}^{*}\left(\mathbf{r}^{\prime}\right) \phi_{b \uparrow}(\mathbf{r})+N_{b} / 2 \phi_{b \downarrow}^{*}\left(\mathbf{r}^{\prime}\right) \phi_{b \downarrow}(\mathbf{r}) .
\end{aligned}
$$


Therefore, all atoms of species $a$ form a fragmented condensate while all atoms of species $b$ form another one. There is one-particle off-diagonal long-range order (ODLRO) within each species.

Note that in our definition of the field operators and reduced density matrices, we have included summation over the two spin states, i.e. we have used a spinor field operator, in considering the coherence between the two spin states. The reduced density matrix of each species characterize the position order for all the atoms of this species.

The interspecies entanglement leads to nonvanishing two-particle reduced density matrix of two particles of different species,

$$
\left\langle G_{S_{z}}\left|\hat{\psi}_{b}^{\dagger}\left(\mathbf{r}_{b}^{\prime}\right) \hat{\psi}_{a}^{\dagger}\left(\mathbf{r}_{a}^{\prime}\right) \hat{\psi}_{a}\left(\mathbf{r}_{a}\right) \hat{\psi}_{b}\left(\mathbf{r}_{b}\right)\right| G_{S_{z}}\right\rangle
$$

which has more than four eigenvalues of order $N^{2}$. Hence there is ODLRO in the interspecies two-particle density matrix. Moreover, there is phase coherence in the interspecies pairs.

The state (5) is the so-called BEC with an entangled order parameter, simply called entangled BEC. Note that there is ODLRO in the single particle density matrix of each species of atoms.

Persistence of entangled BEC. - Away from the symmetric point in the parameter space, the symmetry breaking perturbation $\mathcal{H}_{1}$ becomes nonvanishing. $\mathcal{H}$ still conserves $S_{z}$, though $S$ is no longer conserved. Let us focus on the sector of $S_{z}=0$, in which any eigenstate of $\mathcal{H}$ can be expanded as

$$
\left|\Psi_{n}\right\rangle=\sum_{S} \psi_{n}(S)|S, 0\rangle
$$

where the summation is over $S$, running from $S_{\min } \equiv$ $\left|S_{a}-S_{b}\right|$ to $S_{a}+S_{b}$.

$\mathcal{H}\left|\Psi_{n}\right\rangle=E_{n}\left|\Psi_{n}\right\rangle$ leads to where $N \equiv\left(N_{a}+N_{b}\right) / 2$, $E^{(0)}(S)=K_{e} S(S+1)$. In deriving this equation, we have made use of the following consideration. In the sum in (6), significant contributions come from $S_{\min } \leq S \ll N$ in order to minimize the energy, of which $E^{(0)}(S)$ is the dominant part. In this range of $S$, it can be obtained that $\left\langle S_{a} S_{b} S 0\left|S_{a}^{z}\right| S_{a} S_{b} S 0\right\rangle \approx \delta_{S, S^{\prime}-1} N / 4+\delta_{S, S^{\prime}+1} N / 4$, $\left\langle S_{a} S_{b} S 0\left|S_{b}^{z}\right| S_{a} S_{b} S 0\right\rangle \approx-\delta_{S, S^{\prime}-1} N / 4-\delta_{S, S^{\prime}+1} N / 4$, and $\left\langle S_{a} S_{b} S 0\left|\left(S_{i}^{z}\right)^{2}\right| S_{a} S_{b} S 0\right\rangle \approx \delta_{S^{\prime}, S+2} N^{2} / 16+\delta_{S^{\prime}, S} N^{2} / 8+$ $\delta_{S^{\prime}, S-2} N^{2} / 16$.

In the continuum limit, $\psi_{n}(S+1)+\psi_{n}(S-1)-2 \psi_{n}(S)=$ $\left[\psi_{n}(S+2)+\psi_{n}(S-2)-2 \psi_{n}(S)\right] / 4=d^{2} \psi_{n}(S) / d S^{2}$. Therefore one obtains

$$
-\frac{1}{2} \frac{d^{2} \psi_{n}(S)}{d S^{2}}+\frac{1}{2} \omega^{2} S^{2} \psi_{n}(S)=\nu_{n} \psi_{n}(S),
$$

where $\omega^{2} \equiv 2 K_{e} / d, \nu_{n} \equiv\left(E_{n}+g\right) / d$, with $d=\left(B_{b}-\right.$ $\left.B_{a}\right) N / 2-\left(C_{a}+C_{b}+K_{e}-J_{z}\right) N^{2} / 2, g=\left(B_{b}-B_{a}\right) N / 2-$ $\left(C_{a}+C_{b}+K_{e}-J_{z}\right) N^{2} / 4$. If $\omega^{2}>0$, Eq. (17) becomes the Schrödinger equation for a simple harmonic oscillator, whose $n$-th eigenvalue is $\nu_{n}=\left(n+\frac{1}{2}\right) \omega$. Hence the energy level of the original Hamiltonian (4) is given by

$$
E_{n}=(n+1 / 2) \sqrt{2 K_{e} d}-g .
$$

The interaction gives rise to a term squared in the summation variable. This is the reason why the superposition coefficients behave like eigenfunctions of harmonic oscillators.

Therefore the energy gap of the Hamiltonian (4) is

$$
\Delta=\sqrt{2 K_{e} d}
$$

Thus

$$
\omega=2 K_{e} / \Delta
$$

The ground state of $\mathcal{H}$, in $S_{z}=0$ sector, is thus

$$
\left|\Psi_{0}\right\rangle=\sum_{S} \psi_{0}(S)|S, 0\rangle,
$$

where

$$
\psi_{0}(S)=\mathcal{A} e^{-\frac{1}{2} \omega S^{2}}=\mathcal{A} e^{-\frac{K_{e}}{\Delta} S^{2}},
$$

where the normalization constant $\mathcal{A}=$ $\sqrt{\sqrt{\omega} / \operatorname{erfc}\left(\sqrt{\omega} S_{\min }\right)}$, with $\operatorname{erfc}(x) \equiv \int_{x}^{\infty} e^{-x^{2}} d x$. As $\psi_{0}(S)$ is Gaussian, $S_{\text {min }}$ term dominates. The smaller the energy gap $\Delta$, the more dominant $S_{\text {min }}$ term. Especially, in case $N_{a}=N_{b}, S_{\text {min }}=0$, thus

$$
\psi_{0}(S)=\left(\frac{4 \omega}{\pi}\right)^{1 / 4} e^{-\frac{1}{2} \omega S^{2}}=\left(\frac{8 K_{e}}{\pi \Delta}\right)^{1 / 4} e^{-\frac{K_{e}}{\Delta} S^{2}} .
$$

Hence

$$
\psi_{0}(S) \rightarrow \delta(S), \text { as } \Delta \rightarrow 0
$$

Therefore, in case $N_{a}=N_{b}$, when the energy gap $\Delta$ is vanishing while $K_{e} \propto 1 / \Omega$ remains finite, the ground state of the asymmetric Hamiltonian approaches the symmetric state $\left|S=0, S_{z}=0\right\rangle$. One can say that the symmetry and the entangled BEC is protected by the vanishing energy gap.

More generally, no matter whether $N_{a}=N_{b}$, as far as $\Delta \ll K_{e}$, the ground state of the system can be well approximated as $\left|S_{\text {min }}, 0\right\rangle$. Therefore, the entangled BEC persists as the ground state of $\mathcal{H}$, despite that the symmetry is broken in the Hamiltonian.

A key factor leading to this result is that $\Omega$ remains finite. If, on the contrary, one takes thermodynamic limit $N \rightarrow \infty, \Omega \rightarrow \infty$ while $N / \Omega$ remains constant, then $K_{e} \rightarrow 0$, consequently $\psi_{0}(S)$ becomes independent of $S$, even though one takes $\Delta \rightarrow 0$ after taking $\Omega \rightarrow \infty$. Consequently the ground state becomes an equal superposition of $|S, 0\rangle$ of all possible values of $S$, hence breaks the symmetry. When the symmetry breaking perturbation is infinitesimal, the situation becomes spontaneous symmetry breaking (SSB), and is related to Lieb-Mattis infiniterange model [6], with each species of atoms in our model corresponding to a sublattice in the latter. 


$$
\left(B_{a}-B_{b}\right) \frac{N}{4}\left[\psi_{n}(S-1)+\psi_{n}(S+1)\right]+\left(C_{a}+C_{b}-J_{z}+K_{e}\right) \frac{N^{2}}{16}\left[\psi_{n}(S-2)+2 \psi_{n}(S)+\psi_{n}(S+2)\right]=\left[E_{n}-E^{(0)}(S)\right] \psi_{n}(S),
$$

The present case of persistence of symmetry, and thus fragmentation and entanglement, in a finite-volume condensate, could be viewed as a converse case of SSB of the ground state of an infinite system. Both are due to the near degeneracy of the ground state and the low lying excited states [7]. For SSB, the limit $\Omega \rightarrow \infty$ should be taken before $\Delta \rightarrow 0$. Likewise, for symmetry persistence discussed here, $\Omega$ should be kept finite before taking $\Delta \rightarrow 0$.

Consistency of the isotropic parameter point with the generalized Gross-Pitaevskii equations. - The effective parameters $K$ 's are dependent on the orbital wave functions $\phi_{i \sigma}$ 's, as indicated in Eq. (3). These wave functions satisfy the four generalized Gross-Pitaevskii equations, which are in turn derived from the unique ground state $\left|G_{0}\right\rangle$ at the isotropic parameter point [1]. The equation for $a$ atoms with spin $\sigma$ is $\left\{-\frac{\hbar^{2}}{2 m_{a}} \nabla^{2}+U_{a \sigma}(\mathbf{r})+[2(N-\right.$ 1) $/ 3] g_{\sigma \sigma}^{(a a)}\left|\phi_{a \sigma}(\mathbf{r})\right|^{2}+[(N-1) / 3] g_{\sigma \bar{\sigma}}^{(a a)}\left|\phi_{a \bar{\sigma}}(\mathbf{r})\right|^{2}+[(N-$ 1) $\left./ 3] g_{\sigma \sigma}^{(a b)}\left|\phi_{b \sigma}(\mathbf{r})\right|^{2}+[(2 N+1) / 3] g_{\sigma \bar{\sigma}}^{(a b)}\left|\phi_{b \bar{\sigma}}(\mathbf{r})\right|^{2}\right\} \phi_{a \sigma}(\mathbf{r})-$ $[(N+2) / 6] g_{e} \phi_{b \bar{\sigma}}^{*}(\mathbf{r}) \phi_{b \sigma}(\mathbf{r}) \phi_{a \bar{\sigma}}(\mathbf{r})=\mu_{a \sigma} \phi_{a \sigma}(\mathbf{r})$, where $\bar{\sigma} \neq$ $\sigma, \mu_{a \sigma}$ is the corresponding chemical potential 1 1, each $g$ parameter is the part preceding the integral in the definition of the corresponding $K$ parameter in Eq. (3), i.e. $g_{\sigma \sigma^{\prime}}^{(i j)}=2 \pi \hbar^{2} \xi_{\sigma \sigma^{\prime}}^{(i j)} / \mu_{i j}, g_{e}=4 \pi \hbar^{2} \xi_{e}^{(a b)} / \mu_{a b}$, where $\xi_{e}^{(a b)}=\xi_{\uparrow \downarrow \uparrow \downarrow}^{(a b)}=\xi_{\downarrow \uparrow \uparrow \downarrow}^{(a b)}$. The equation for $\phi_{b \sigma}(\mathbf{r})$ is in a similar form.

Therefore, in order to attain the isotropic parameter point, both the orbital wave functions and the scattering lengths need to be constrained in order that the effective parameters satisfy the requirements that $B_{i}=C_{i}=0$ and $J_{z}=K_{e}$. Because the generalized Gross-Pitaevskii equations, which govern the orbital wave functions, are in turn derived at the isotropic parameter point, a problem arises whether these requirements are consistent with the generalized Gross-Pitaevskii equations.

Here we show that such consistency is indeed guaranteed under the following conditions. (i) $U_{i \uparrow}(\mathbf{r})=U_{i \downarrow}(\mathbf{r})$. (ii) The intraspecies scattering lengths $\xi_{\sigma_{1} \sigma_{2} \sigma_{3} \sigma_{4}}^{(i i)}$ 's, for physically allowed channels, are all equal for each species $i$, denoted as $\xi_{i}$. Note that this means $\xi_{\sigma \bar{\sigma}}^{(i i)}=2 \xi_{\sigma \sigma}^{(i i)}=2 \xi_{i}$ in shorthands. (iii) The interspecies scattering lengths satisfy the relations $\xi_{\uparrow \uparrow}^{(a b)}=\xi_{\downarrow \downarrow}^{(a b)}$, denoted as $\xi_{s}^{(a b)}$, and $\xi_{\uparrow \downarrow}^{(a b)}=\xi_{\downarrow \uparrow}^{(a b)}$, denoted as $\xi_{d}^{(a b)}$, where the subscripts "s" and "d" represent "same" and "different", respectively.

First, by considering that the nonlinear terms of interaction vanish at the boundary of the condensate, it can be

\footnotetext{
${ }^{1}$ In Ref. [1], as a Lagrange multiplier for the normalization of $\phi_{a \sigma}, \mu_{a \sigma}$ represents $N / 2$ multiplied by the chemical potential
}

seen that $U_{i \uparrow}(\mathbf{r})=U_{i \downarrow}(\mathbf{r})$ implies $\mu_{i \uparrow}=\mu_{i \downarrow}$. Then, under the above three conditions, in considering that as a wave function of bosons, $\phi_{i \sigma}$ is real, one obtains, from the difference of the generalized Gross-Pitaevskii equations for $\phi_{a \uparrow}$ and $\phi_{a \downarrow}$,

$\left(\frac{N-1}{3} g_{s}-\frac{2 N+1}{3} g_{d}\right)\left(\frac{\phi_{b \uparrow}}{\phi_{b \downarrow}}-\frac{\phi_{b \downarrow}}{\phi_{b \uparrow}}\right)=\frac{N+2}{6} g_{e}\left(\frac{\phi_{a \uparrow}}{\phi_{a \downarrow}}-\frac{\phi_{a \downarrow}}{\phi_{a \uparrow}}\right)$,

where $g_{s / d}=2 \pi \hbar^{2} \xi_{s / d}^{(a b)} / \mu_{a b}$. Similarly,

$\left(\frac{N-1}{3} g_{s}-\frac{2 N+1}{3} g_{d}\right)\left(\frac{\phi_{a \uparrow}}{\phi_{a \downarrow}}-\frac{\phi_{a \downarrow}}{\phi_{a \uparrow}}\right)=\frac{N+2}{6} g_{e}\left(\frac{\phi_{b \uparrow}}{\phi_{b \downarrow}}-\frac{\phi_{b \downarrow}}{\phi_{b \uparrow}}\right)$.

Consequently, we obtain $\phi_{a \uparrow}=\phi_{a \downarrow}$ and $\phi_{b \uparrow}=\phi_{b \downarrow}$, unless $(N-1) g_{s}=(2 N+1) g_{d}$. Subsequently, $\epsilon_{i \uparrow}=\epsilon_{i \downarrow}$ according to Eq. (2). Consequently, $B_{i}=C_{i}=0$ if the scattering lengths satisfy the latter two conditions above. Moreover, $J_{z}=K_{e}$ if $\xi_{e}^{(a b)}=\xi_{s}^{(a b)}-\xi_{d}^{(a b)}$.

Transition Temperature. - We briefly discuss the issue of transition temperature. A crude estimation can be made by following the idea of Ashhab and Leggett on $S U(2)$ symmetric model of one species of spin- $\frac{1}{2}$ atoms [8]. One may consider four independent interpenetrating gases of $N_{i \sigma}$ atoms of species $i$ with spin $\sigma,(i=a, b, \sigma=\uparrow$ $, \downarrow)$. The transition temperature for each gas is $T_{i \sigma} \approx$ $3.31 \hbar^{2} m\left(N_{i \sigma} / V\right)^{2 / 3}$. The error due to ignoring spin exchanges is of the order of $\sqrt{N}$, and can be neglected in this estimation. Hence the transition temperature of the entangled BEC is roughly the minimum among $T_{i \sigma}$ 's. Below this temperature, the noncondensed atoms of each species with each spin is $\approx\left(m T / 3.31 \hbar^{2}\right)^{3 / 2}$

Experimental implementations. - We now consider how to implement this model by using the trapped alkali atoms. In order to constrain each atom in the Hilbert space of only two spin states, scattering to other spin states must be suppressed.

We propose to represent the two pseudospin states as the hyperfine states $\left|F=2, m_{F}=2\right\rangle$ and $\left|F=1, m_{F}=1\right\rangle$ of an alkali atom with nuclear spin $I=3 / 2\left({ }^{7} \mathrm{Li},{ }^{23} \mathrm{Na}\right.$, ${ }^{39} \mathrm{~K},{ }^{41} \mathrm{~K}$ and $\left.{ }^{87} \mathrm{Rb}\right)$. Similarly, one can also use $\mid F=$ $\left.2, m_{F}=-2\right\rangle$ and $\left|F=1, m_{F}=-1\right\rangle$ when the magnetic field is small so that their energy difference is large. Because of the large energy splitting between the two hyperfine states [9], the number of atoms with $F=2$ is forbidden to increase in each scattering. Furthermore, in an energetically allowed scattering channel, conservations of the total $F$ and $m_{F}$ of the two atoms guarantee that the hyperfine states of the two scattered atoms are either unchanged or exchanged, just as in our model. Besides, because of its various advantages, e.g. large interspecies scattering lengths [10], a mixture of isotopes 
${ }^{85} \mathrm{Rb}$ and ${ }^{87} \mathrm{Rb}$ can also be used. For ${ }^{85} \mathrm{Rb}, I=5 / 2$, consequently $\left|F=3, m_{F}=3\right\rangle$ and $\left|F=2, m_{F}=2\right\rangle$ play similar roles as $\left|F=2, m_{F}=2\right\rangle$ and $\left|F=1, m_{F}=1\right\rangle$ of atoms with $I=3 / 2$, respectively. Thus these two sets of hyperfine states can represent the two pseudospin states of the two species in our model. Similarly, one can use $\left|F=3, m_{F}=-3\right\rangle$ and $\left|F=2, m_{F}=-2\right\rangle$ of ${ }^{85} \mathrm{Rb}$ while $\left|F=2, m_{F}=-2\right\rangle$ and $\left|F=1, m_{F}=-1\right\rangle$ of ${ }^{87} \mathrm{Rb}$. The number of ${ }^{85} \mathrm{Rb}$ cannot be too large in order to avoid collapse, as its intraspecies scattering length is negative.

Magnetic trapping implementation is not favored for the following reasons. First of all, only one of the two hyperfine states representing the two pseudospin states is lowfield seeker and can be trapped. Besides, one of the above conditions for the consistency of isotropic parameter point, namely $U_{i \uparrow}=U_{i \downarrow}$, cannot be satisfied, because the magnetic trapping potential is proportional to $g_{F} m_{F}[9]$, hence has different values for the two hyperfine states. Moreover, it is difficult to tune the scattering lengths in a magnetic trap.

In contrast, the implementation can be made in an optical trap, where the trapping potential is based on the single-atom energy shift due to red detuning in coupling with the laser, and is independent of atomic spins [9], thus the two hyperfine states representing the pseudospin states can both be trapped. Moreover, $U_{i \uparrow}=U_{i \downarrow}$ is satisfied. Furthermore, the scattering lengths can be tuned by using Feshbach resonances. Therefore, the consistency of the isotropic parameter point can be achieved. Even though the effective parameters deviate from the exact isotropic point, the energy gap can still be tuned to vanish, so that the ground state still approaches the entangled BEC.

Let us summarize some relevant experiences obtained from previous experiments on BEC mixtures. In general, for $\mathrm{BEC}$ in optical traps, atoms are first prepared in a magnetic trap. Optical pumping and adiabatic passage or radiofrequency sweep are often used in preparing atoms in certain spin states by transferring atoms from other spin states. These methods can be adopted in preparing our system. In the early experiments in magnetic traps, on mixtures of $\left|F=2, m_{F}=2\right\rangle$ and $\left|F=1, m_{F}=-1\right\rangle$ of ${ }^{87} \mathrm{Rb}[11]$ and of $\left|F=2, m_{F}=1\right\rangle$ and $\left|F=1, m_{F}=-1\right\rangle$ of ${ }^{87} \mathrm{Rb}[12]$, spin exchange scattering led to atom loss from the magnetic trap. BEC with multiple components of different spin states were studied in the optically trapped ${ }^{23} \mathrm{Na}$ with $F=1$ [13] and ${ }^{87} \mathrm{Rb}$ with $F=1$ [14], where spin exchange scattering indeed did not produce atoms with $F=2$. Spin exchange scattering were also observed in ${ }^{87} \mathrm{Rb}$ with $F=2$ in optical traps, where in some cases, e.g. the two initial hyperfine states are $\mid F=2, m_{F}=$ $2\rangle$ and $\left|F=2, m_{F}=-2\right\rangle$, or both $\left|F=2, m_{F}=0\right\rangle$, scattering between $F=2$ atoms lead to $F=1$ atoms [15]. But such $F=2$ to $F=1$ scattering is prohibited by the conservation of total $m_{F}$ if the initial hyperfine states are $\left|F=2, m_{F}=2\right\rangle$ and $\left|F=2, m_{F}=1\right\rangle$, or both $\left|F=2, m_{F}=2\right\rangle$, as in our proposed implementations.

Two experimental systems of BEC mixtures are very close to, and might be extended to, the realization of entangled BEC. One is a mixture of ${ }^{41} \mathrm{~K}$ and ${ }^{87} \mathrm{Rb}$, which was regarded as the most favorable candidate for realizing BEC mixture [16]. Their BEC mixture has been realized in a magnetic trap, with both species in $\mid F=2, m_{F}=$ 2) [17], as well as in an optical trap, with both species in $\left|F=1, m_{F}=1\right\rangle[18]$. In preparing the latter experiment, atoms are transferred to $\left|F=1, m_{F}=1\right\rangle$ from $\left.F=2, m_{F}=2\right\rangle$ by applying a microwave and a radiofrequency sweep. This procedure could also be used for our purpose, with only half of the atoms transferred. Interspecies Feshbach resonances, as needed also for our system, were used in this experiment. The other experimental system close to ours is that of ${ }^{85} \mathrm{Rb}$ in $\left|F=2, m_{F}=-2\right\rangle$ and ${ }^{87} \mathrm{Rb}$ in $\left|F=1, m_{F}=-1\right\rangle$, in which Feshbach resonances were used in creating heteronuclear molecules [19] and in tuning the two-species BEC [20]. To realize entangled BEC, we need also ${ }^{85} \mathrm{Rb}$ in $\left|F=3, m_{F}=-3\right\rangle$ and ${ }^{87} \mathrm{Rb}$ in $\left|F=2, m_{F}=-2\right\rangle$, which can be transferred from the other spin state of each isotope by optical pumping or radiofrequency sweep.

As an alternative approach, the two pseudospin states could also be implemented by using atoms with negligible hyperfine coupling [21].

To prepare an entangled BEC, one may first prepare four condensates of equal numbers of atoms, of the two species with the two spin states, as prescribed above. Then conservations of the total spin and its $z$-component of any two scattered atoms dictate that these atoms remain in the manifold of these spin states. The interaction between these four condensates builds up coherence among them, and realizes entangled BEC of the total system.

Summary. - To summarize, we have examined the feasibility of experimental realization of the entangled $\mathrm{BEC}$, i.e. BEC occurring in an interspecies entangled two-particle state, as the ground state of a model of two species of atoms with spins, proposed in Ref. [1]. The entangled BEC is a novel type of fragmented BEC. We have analytically shown that in a wide parameter regime, the entangled BEC persists as the ground state of the concerned model, as far as the energy gap tends to vanish. This makes the entangled BEC more accessible in experiments. Subsequently, we established the consistency between the isotropic point of the effective parameters and the generalized Gross-Pitaevskii equations, which govern the orbital wave functions, on which the effective parameters depend. A brief estimation of the transition temperature was made. Finally, we discussed how to experimentally realize this model with the entangled $\mathrm{BEC}$ as its ground state. We found that it is suitable to use an optical trap. Using two species of atoms with $I=3 / 2$, the two pseudospin states of the two species can be realized both in terms of $\left|F=2, m_{F}=2\right\rangle$ and $\left|F=1, m_{F}=1\right\rangle$, or both in terms of $\left|F=2, m_{F}=-2\right\rangle$ and $\left|F=1, m_{F}=-1\right\rangle$. In view of previous experiments, a most favorable candidate is the mixture of ${ }^{41} \mathrm{~K}$ and ${ }^{87} \mathrm{Rb}$. Another candidate is the mixture of ${ }^{87} \mathrm{Rb}$ and ${ }^{85} \mathrm{Rb}$. For 
${ }^{85} \mathrm{Rb}, I=5 / 2$, thus the two pseudospin states are represented by $\left|F=3, m_{F}=3\right\rangle$ and $\left|F=2, m_{F}=2\right\rangle$ when $\left|F=2, m_{F}=2\right\rangle$ and $\left|F=1, m_{F}=1\right\rangle$ are used for ${ }^{87} \mathrm{Rb}$, or by $\left|F=3, m_{F}=-3\right\rangle$ and $\left|F=2, m_{F}=-2\right\rangle$ when $\left|F=2, m_{F}=-2\right\rangle$ and $\left|F=1, m_{F}=-1\right\rangle$ are used for ${ }^{87} \mathrm{Rb}$.

I thank Rukuan Wu for discussions. This work is supported by National Science Foundation of China (Grant No. 10674030) and Shuguang Project (Grant No. 07S402).

\section{REFERENCES}

[1] Y. Shi and Q. Niu, Phy. Rev. Lett. 96, 140401 (2006).

[2] P, Noziers, in Bose-Einstein Condensation, edited by A. Griffin, D. W. Snoke and S. Stringari (Cambridge University Press, Cambridge, 1995).

[3] M. Koashi and M. Ueda, Phy. Rev. Lett. 84, 1066 (2000); T. L. Ho and S. K. Yip, Phy. Rev. Lett. 84, 4031 (2000).

[4] E. J. Mueller, T. L. Ho, M. Ueda and G. Baym, Phys. Rev. A 74, 033612 (2006) and references therein.

[5] A. J. Leggett, Rev. Mod. Phys. 73, 307 (2001).

[6] E. Lieb and D. Mattis, J. Math. Phys. (N.Y.) 3, 749 (1962); C. Kaiser and I. Peschel, J. Phys. A 22, 4257 (1989); T. A. Kaplan, W. von der Linden and P. Horsch, Phys. Rev. B 42, 4663 (1990); J. van Wezel, J. Zaanen and J. van den Brink, Phys. Rev. B 74, 094430 (2006).

[7] P. W. Anderson, Basic Notions in Condensed Matter Physics (Benjamin/Cummings, Menlo Park, 1984); A. J. Leggett, in Ref. [2]; S. Weinberg, Quantum Theory of Fields II (Cambridge University Press, Cambridge, 1996).

[8] S. Ashhab and A. J. Leggett, Phys. Rev. A 65, 023604 (2002).

[9] C. J. Pethick and H. Smith, Bose-Einstein Condensation in Dilute Gases (Cambridge University Press, Cam bridge, 2002).

[10] J. P. Burke et al., Phy. Rev. Lett. 80, 2097 (1998).

[11] C. J. Myatt, et al., Phy. Rev. Lett. 78, 586 (1997).

[12] D. S. Hall et al., Phy. Rev. Lett. 81, 1539 (1998); D. S. Hall et al., Phy. Rev. Lett. 81, 1543 (1998).

[13] J. Stenger et al., Nature 396, 345 (1998); H.-J. Miesner et al., Phy. Rev. Lett. 82, 2228 (1999); D. M. Stamper-Kurn et al., Phy. Rev. Lett. 83, 661 (1999).

[14] M. S. Chang et al., Nature Phys. 1, 111 (2005); L. E. Sadler et al., Nature 443, 312 (2006).

[15] H. Schmaljohann et al., Phy. Rev. Lett. 92, 040402 (2004); M. S. Chang et al., Phy. Rev. Lett. 92, 140403 (2004); T. Kuwamoto et al., Phy. Rev. A 69, 063604 (2004).

[16] G. Ferrari et al., Phy. Rev. Lett. 89, 053202 (2002).

[17] G. Modugno et al., Phy. Rev. Lett. 89, 190404 (2002).

[18] G. Thalhammer et al., Phy. Rev. Lett. 100, 210402 (2008).

[19] S. B. Papp, J. M. Pino and C. E. Wieman, Phy. Rev. Lett. 97, 180404 (2006).

[20] S. B. Papp, J. M. Pino and C. E. Wieman, Phy. Rev. Lett. 101, 040402 (2008).

[21] R. Hulet, private communication. 